\title{
Effects of Root Debridement With Hand Curettes and Er:YAG Laser on Chemical Properties and Ultrastructure of Periodontally-Diseased Root Surfaces Using Spectroscopy and Scanning Electron Microscopy
}

\author{
Reza Amid ${ }^{1,2}$, Gholam Ali Gholami ${ }^{1}$, Masoud Mojahedii ${ }^{3}$,Maryam Aghalou ${ }^{1}$, Mohsen Gholami ${ }^{4}$, Mahdieh \\ Mirakhori $^{*}$ \\ 'Deptartment of Periodontics, School of Dentistry, Shahid Beheshti University of Medical Sciences, Tehran, Iran \\ ${ }^{2}$ Dental Research Center, Research Institute of Dental Sciences, School of Dentistry, Shahid Beheshti University of Medical \\ Sciences, Tehran, Iran \\ ${ }^{3}$ Deptartmen of Laser, School of Dentistry, Shahid Beheshti University of Medical Sciences, Tehran, Iran \\ ${ }^{4}$ Private Practice, Tehran, Iran
}

\section{*Correspondence to \\ Mahdieh Mirakhori, M.D; Gifted and Talented Dental Students \\ Division, Students Research \\ Committee, School of dentistry, Shahid Beheshti University of Medical Sciences, Tehran, Iran. Tel: +98-9193919987; \\ Email: \\ Mirakhori_mahdie@yahoo.com}

Published online 28 March 2017

\begin{abstract}
Introduction: The efficacy of erbium-doped yttrium aluminum garnet (Er:YAG) laser for root debridement in comparison with curettes has been the subject of many recent investigations. Considering the possibility of chemical and ultra-structural changes in root surfaces following laser irradiation, this study sought to assess the effects of scaling and root planing (SRP) with curettes and Er:YAG laser on chemical properties and ultrastructure of root surfaces using spectroscopy and scanning electron microscopy (SEM).

Methods: In this in vitro experimental study, extracted sound human single-rooted teeth $(n=50)$ were randomly scaled using manual curettes alone or in conjunction with Er:YAG laser at 100 and $150 \mathrm{~mJ} /$ pulse output energies. The weight percentages of carbon, oxygen, phosphorous and calcium remaining on the root surfaces were calculated using spectroscopy and the surface morphology of specimens was assessed under SEM. Data were analyzed using one-way analysis of variance (ANOVA).

Results: No significant differences $(P>0.05)$ were noted in the mean carbon, oxygen, phosphorous and calcium weight percentages on root surfaces following SRP using manual curettes with and without laser irradiation at both output energies. Laser irradiation after SRP with curettes yielded rougher surfaces compared to the use of curettes alone.

Conclusion: Although laser irradiation yielded rougher surfaces, root surfaces were not significantly different in terms of chemical composition following SRP using manual curettes with and without Er:YAG laser irradiation. Er:YAG laser can be safely used as an adjunct to curettes for SRP.

Keywords: Absorption spectroscopy; Nonsurgical periodontal treatment; Erbium YAG Laser; Electron scanning microscopy.
\end{abstract}

\section{Introduction}

Periodontal diseases are among the most common infectious diseases caused by the accumulation of bacterial plaque on tooth surfaces. Periodontitis can result in soft and hard tissue destruction and eventual tooth loss. Scaling and root planning with hand instruments, sonic and ultrasonic devices and air abrasion are performed for treatment of periodontal diseases. ${ }^{1}$ Nonsurgical periodontal debridement is performed aiming to efficiently eliminate plaque and calculus without damaging root surfaces. Manual instruments and ultrasonic devices have long been used for scaling and root planing (SRP) with successful results. ${ }^{2}$

Dental lasers were recently introduced to increase the efficacy of conventional SRP, and many studies have evaluated their efficacy in enhancing the biocompatibility and attachment of fibroblasts to root surfaces. ${ }^{3}$ The efficacy of erbium-doped yttrium aluminum garnet (Er:YAG) laser

Please cite this article as follows: Amid R, Gholami GA, Mojahedi M, Aghalou A, Gholami M, Mirakhori M. Effects of root debridement with hand curettes and Er:YAG laser on chemical properties and ultrastructure of periodontally-diseased root surfaces using spectroscopy and scanning electron microscopy. J Lasers Med Sci. 2017;8(2):66-71. doi:10.15171/jlms.2017.12. 
with $120 \mathrm{~mJ} /$ pulse output energy was evaluated in a previous study and it was shown that although Er:YAG laser produced smoother surfaces than curettes, some irregular surface areas and craters were still present on the root surfaces. ${ }^{4}$ Thus, some researchers have recommended lasers with lower output energies to prevent the unwanted physicochemical side effects (i.e. carbonization). ${ }^{5}$ Considering these shortcomings, soft tissue lasers were introduced, which are incapable of removing hard tissues. ${ }^{6,7}$ Diode lasers are among the commonly used soft tissue lasers. These lasers are not much efficient for calculus removal; but can be used as an adjunct to conventional SRP to enhance its efficacy in yielding biocompatible and germ-free root surfaces. ${ }^{8}$

Laser irradiation causes significant chemical changes in root surfaces. Weight percentages of carbon, phosphorous, oxygen and calcium are particularly important in this regard. No previous study has quantitatively assessed chemical properties of root surfaces after the application of Erbium laser. Thus, this study aimed to compare the effects of hand curettes and Er:YAG laser on chemical properties and ultrastructure of periodontally-diseased root surfaces using spectroscopy and scanning electron microscopy (SEM).

\section{Methods}

This in vitro experimental study was conducted on 50 (80\% power at the 0.05 level of significance) sound single-rooted maxillary and mandibular teeth freshly extracted due to periodontal disease or orthodontic reason. The selected teeth were sound with no carious lesions, fractures, or cracks. To ensure the absence of any defect, root surfaces were evaluated at $4 \mathrm{X}$ magnification using a magnifier. Teeth surfaces were polished using prophylactic paste and bristle brush and stored in phosphate buffered solution (PBS). The teeth randomly underwent one of the following treatment modalities:

Experimental group 1: Surfaces of 10 roots were scaled using \#7 and \#8 Hand curettes (Nordent, IL, USA) until a smooth surface was achieved. Surface smoothness was determined by the operator using the sharp tip of an explorer.

Experimental group 2: Surfaces of 10 roots were debrided using hand curettes and were then irradiated with Er:YAG laser with $100 \mathrm{~mJ} /$ pulse output energy, $15 \mathrm{~Hz}, 50 \%$ air and $85 \%$ water at $60^{\circ}$ angle for 20 seconds.
Experimental group 3: Surfaces of 10 roots were scaled using hand curettes and were then irradiated with Er:YAG laser with $150 \mathrm{~mJ} /$ pulse output energy, $15 \mathrm{~Hz}, 50 \%$ air and $85 \%$ water at $60^{\circ}$ angle for 20 seconds.

Negative control group: Ten roots with bacterial debris and calculus were evaluated as the negative controls.

Positive control group: Ten roots without calculus and bacterial debris were evaluated as positive controls.

The percentage of main elements remained on root surfaces including carbon, oxygen, calcium and phosphorous was determined using spectroscopy with $\mathrm{x}$-ray diffraction (XRD) and $\mathrm{x}$-ray fluorescent (XRF) techniques. XRF analyzer spectrometer is used to measure the percentage of elements in the composition of a material and is a suitable technique for the measurement of the weight percentage of elements.

For SEM analysis, specimens were cut into $2 \mathrm{~mm}$ sections and the sections were dehydrated in acetone in an incubator for 12 hours. Specimens were then dried, gold-coated and evaluated under a SEM (KYKY EM3200, China). The examiner assessing the surface roughness under SEM and the operator performing spectroscopy were blinded to the group allocation of samples and the type of SRP modality. Data were analyzed using SPSS version 16 (SPSS Inc, Chicago, IL, USA). Weight percentages of elements on the root surfaces of specimens in the 5 groups were analyzed using one-way analysis of variance (ANOVA). Considering the lack of a significant difference in the results of one-way ANOVA, pairwise comparison of groups was not performed. Type 1 error was considered as 0.05 and $P<0.05$ was considered statistically significant.

\section{Results}

The mean and standard deviation (SD) weight percentages of carbon, oxygen, phosphorous and calcium remaining on the root surfaces following root debridement with hand curettes alone and in conjunction with Er:YAG laser with 100 and $150 \mathrm{~mJ} /$ pulse output energy are presented in Table 1.

According to the results of one-way ANOVA, no significant difference was found in the mean weight percentages of carbon $(P=0.110)$, oxygen $(P=0.660)$, phosphorous $(P=0.060)$ and calcium $(P=0.080)$ on the root surfaces among the 3 experimental groups.

Root surfaces treated with 150 and $100 \mathrm{~mJ}$ laser irradiation showed the maximum values of carbon and oxygen,

Table 1. The Mean Weight Percentages of Carbon, Oxygen, Phosphorous and Calcium on the Root Surfaces of the 3 Experimental and Positive and Negative Control Groups

\begin{tabular}{|c|c|c|c|c|c|c|c|c|c|c|c|c|}
\hline & \multicolumn{3}{|c|}{ Carbon } & \multicolumn{3}{|c|}{ Oxygen } & \multicolumn{3}{|c|}{ Phosphorous } & \multicolumn{3}{|c|}{ Calcium } \\
\hline & Mean \pm SD & Min & Max & Mean $\pm S D$ & Min & Max & Mean \pm SD & Min & Max & Mean \pm SD & Min & Max \\
\hline Hand curette & $44.96 \pm 20.38$ & 22.65 & 83.29 & $30.63 \pm 14.19$ & 9.76 & 53.2 & $6.23 \pm 3.54$ & 0.51 & 11.24 & $14.86 \pm 7.77$ & 0.88 & 26.94 \\
\hline Laser $100 \mathrm{~mJ}$ & $38.47 \pm 14.99$ & 22.8 & 60.84 & $36.93 \pm 10.17$ & 18.32 & 48.99 & $7.28 \pm 2.08$ & 4.37 & 10.24 & $17.19 \pm 4.79$ & 8.98 & 22.75 \\
\hline Laser $150 \mathrm{~mJ}$ & $53.1 \pm 14.02$ & 29.0 & 76.79 & $33.34 \pm 8.8$ & 17.87 & 48.41 & $4.29 \pm 2.6$ & 0 & 8.37 & $9.35 \pm 5.42$ & 0.49 & 16.33 \\
\hline Control - & $34.52 \pm 8.19$ & 22.77 & 49.04 & $36.48 \pm 6.49$ & 25.05 & 44.41 & $7.74 \pm 2.19$ & 4.85 & 12.01 & $17.54 \pm 7.58$ & 7.36 & 33.57 \\
\hline Control + & $44.6 \pm 18.83$ & 20.49 & 67.76 & $34.47 \pm 10.66$ & 16.33 & 44.4 & $5.37 \pm 3.17$ & 0.44 & 9.87 & $15.02 \pm 8.54$ & 2.62 & 26.32 \\
\hline
\end{tabular}

Abbreviations: Max, maximum; Min, minimum; SD, standard deviation. 
respectively. Maximum and minimum values of calcium and phosphorus detected in negative control and $150 \mathrm{~mJ}$ laser groups, respectively. Qualitative changes of root surfaces treated with different protocols are showed in Figure 1.

\section{Discussion}

Based on the results, no significant difference was noted in weight percentages of carbon, oxygen, phosphorous and calcium remaining on the root surfaces following SRP with manual curettes with and without Er:YAG laser with 100 and $150 \mathrm{~mJ}$ output energies.

In a study by Altundasar et al, in 2006, SEM/EDX analysis of specimens irradiated with laser revealed increased weight percentage of calcium and magnesium; which is in contrast with our findings. ${ }^{9}$ In our study, the weight percentage of carbon in the manual curette $+150 \mathrm{~mJ}$ Er:YAG laser group was slightly (but not significantly) higher than that in the 2 other experimental groups $(53.1 \%$ in the manual curette $+150 \mathrm{~mJ}$ laser versus $44.96 \%$ in the manual curette alone and $38.47 \%$ in the manual curette plus $100 \mathrm{~mJ}$ laser). Also, the weight percentage of calcium in the manual curette $+150 \mathrm{~mJ}$ Er:YAG laser group was lower than that in the 2 other experimental groups (9.35\% versus $14.86 \%$ and $17.19 \%)$. The absence of any significant difference in calcium/phosphorous ratios was indicative of no significant change at the molecular level. During laser irradiation, the evaporation of organic compounds may result in increased calcium or phosphorous content of the superficial dentin. However, our obtained results must be interpreted with caution because SEM/ EDX analyses of weight percentages of elements such as calcium, phosphorous and oxygen have high technical sensitivity. ${ }^{10}$ In the clinical setting, increased content of important elements after laser irradiation may increase the resistance of dentinal surfaces to acid attacks. ${ }^{11}$ On the other hand, it was found that laser irradiation would increase the resistance of teeth to cariogenic agents. ${ }^{12}$
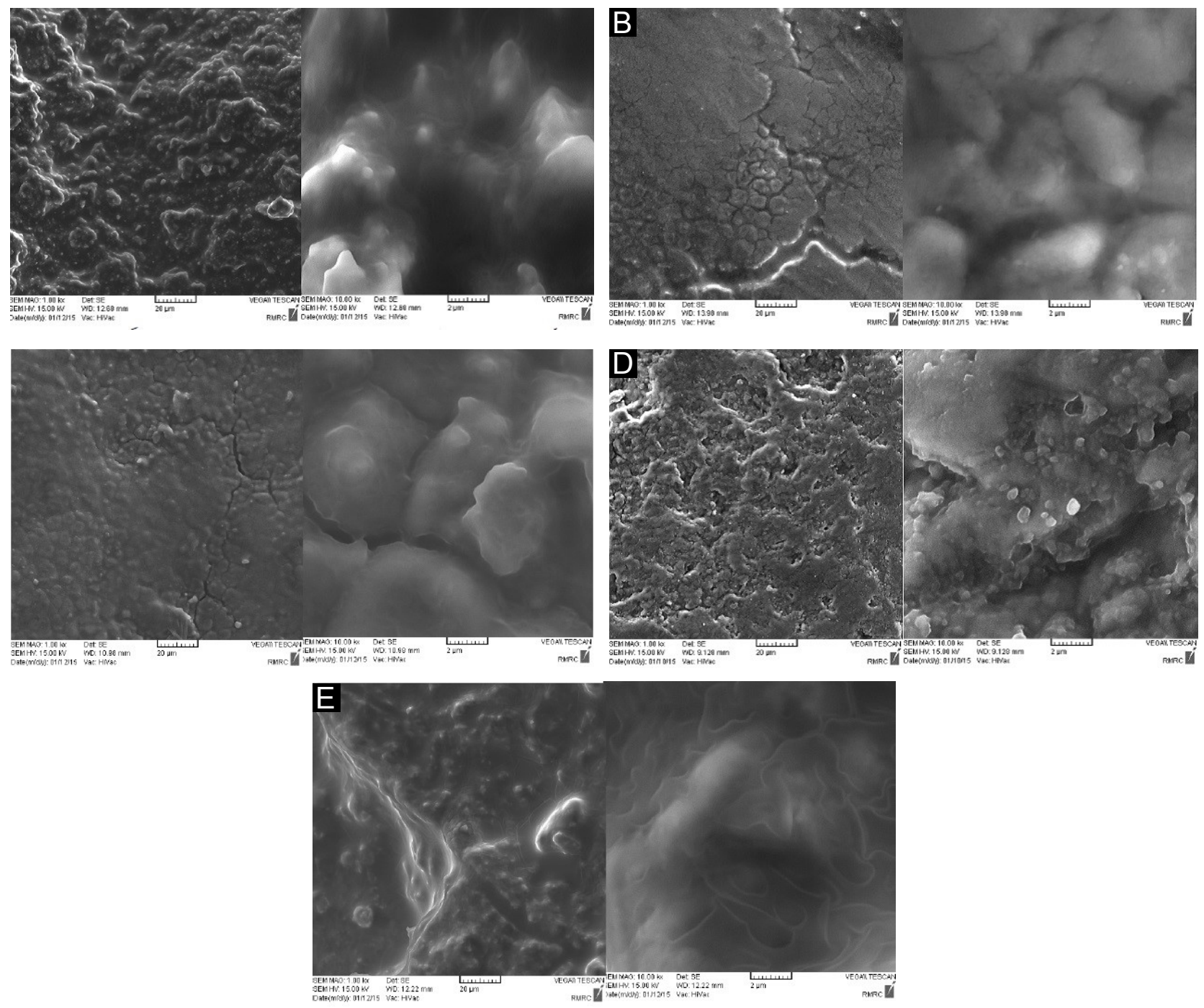

Figure 1. SEM Micrographs of Root Surfaces in (A) the Negative Control Group, (B) the Positive Control Group, (C) the Experimental Group 1 (Hand Curette Alone), (D) the Experimental Group 2 (Hand Curette $+100 \mathrm{~mJ}$ Er:YAG Laser), and (E) the Experimental Group 3 (Hand Curette $+150 \mathrm{~mJ}$ Er:YAG Laser) at Different Magnifications. 
Near-infrared laser wavelengths such as $808 \mathrm{~nm}$ diode laser appear to cause changes in the organic matrix of enamel and confer resistance to demineralization. This is of clinical importance since the preservation of organic matrix in dentin lesions is important for decreasing the risk of caries progression. ${ }^{13}$ In this regard, weight percentages of dentin constituents (70\% hydroxyapatite, $20 \%$ organic matrix and 10\% water), which are different from enamel constituents $(96 \%$ minerals, water and organic matrix), must be taken into account.

To the best of our knowledge, no previous study has compared the chemical changes of root surfaces following SRP with manual curettes and laser irradiation. Thus, the comparison of results was not feasible.

De-Melo et al in 2011 assessed the effect of low intensity diode laser (808 $\mathrm{nm}$ wavelength) on chemical composition of dentin and the prevention of dentin demineralization. EDX analyses of calcium, phosphorous, carbon and oxygen contents in different groups revealed no significant difference; which is similar to our results. ${ }^{14}$ However, in their study, calcium and phosphorous contents slightly decreased post-treatment and significant changes were noted in the results of EDX analysis. Thus, EDX analyses may only be useful for the assessment of specimens containing standard concentrations of chemicals. Moreover, EDX only assesses the presence of trapped molecules and cannot determine the molecular characteristics of specimens. Relative similarity of carbon, oxygen, phosphorous and calcium weight percentages on root surfaces following SRP with manual curettes and 100 and $150 \mathrm{~mJ}$ Er:YAG laser irradiation in our study indicates that these treatment modalities may have equal effects in terms of chemical changes in root surfaces. This finding also confirms the safety of Er:YAG laser. However, these observations must be further scrutinized and confirmed in future studies by the use of methods such as Raman spectroscopy. ${ }^{15}$ Clinically, SRP is performed to achieve smooth and resistant root surfaces, which are important for a healthy periodontium. ${ }^{16,17}$ In the current study, the irradiation of 100 and $150 \mathrm{~mJ}$ Er:YAG laser increased the surface roughness according to SEM observations. Rough surfaces enhance the accumulation of biofilm especially when the rough surfaces are located supragingivally. ${ }^{16}$ Thus, these surfaces must be polished to prevent the accumulation of bacterial biofilm. ${ }^{17}$ On the other hand, subgingival rough surfaces may be beneficial for periodontal treatments because they enhance the adhesion of a stable fibrin network and lead to subsequent migration of fibroblasts and mesenchymal cells. These interactions accelerate the process of periodontal regeneration. ${ }^{18}$

SEM analysis of specimens subjected to laser irradiation (100 and $150 \mathrm{~mJ}$ ) and SRP with manual curettes revealed variable degrees of changes in root surfaces. These results were in accordance with the findings of de Oliveira et al. ${ }^{19}$ They reported that all root surfaces irradiated with erbium, chromium doped yttrium scandium gallium garnet (Er-Cr: YSGG) laser were rougher than non-lased surfaces (similar to our study). However, these observations were all subjective.

Foroutan et al compared the manual tools ultrasonic and Er:YAG laser on the debridement effect of the surface of the root suffering from periodontitis. They showed that although various dental surfaces cleaning methods may be different in other aspects, but are all similar concerning the fibroblasts morphology. Also in addition to the power, laser emission time may also be effective in the cells morphology results. ${ }^{20}$

Also, Amid et al evaluated the effects of manual curettes, ultrasonic scaler and Er:YAG laser on surface roughness and ultrastructure of periodontally-diseased root surfaces and found no significant difference in terms of changes in surface roughness parameters or surface changes among different techniques. However, laser irradiated surfaces were more irregular and experienced greater surface distortion. ${ }^{21}$

In a study by Birang et al, Er:YAG laser caused greater root surface roughness than hand instruments; this finding is in line with the results of the current study. ${ }^{22}$

Schwartz et al showed that the efficacy of calculus removal by Er:YAG laser irradiation in the clinical setting was similar to that of hand instruments; but, diode laser irradiation was not suitable for calculus removal due to significant surface distortion caused. ${ }^{23}$ Frentzen et al reported increased loss of cementum and dentin and high surface roughness due to Er:YAG laser irradiation and questioned its clinical application. ${ }^{24}$ On the other hand, de Mendonca et al in 2008 reported that SRP with manual curettes caused rougher surfaces than Er:YAG laser irradiation and ultrasonic scaler, and added that all methods increased root surface roughness after treatment. ${ }^{4}$ Crespi et al in 2006 indicated that Er:YAG laser irradiation in the clinical setting resulted in plaque and calculus removal and yielded a rough surface morphology. ${ }^{25}$ Tsurumaki et al in 2011 showed that ultrasonic scalers and Er, Cr:YSGG laser irradiation alone or in combination with hand instrumentation yielded in rougher surfaces than SRP with manual curettes. Although the type of laser used in their study was different from ours, their findings were in accord with our results. ${ }^{26}$

SRP is performed to remove dental plaque and calculus from root surfaces of teeth. The remaining root surface roughness after treatment is believed to be less than that prior to treatment. ${ }^{27}$ However, smooth root surfaces are not a perquisite for a successful SRP. ${ }^{28,29}$ Smooth root surfaces at the gingival margin are advantageous because the odds of dental plaque accumulation on smooth surfaces are less than that on rough surfaces and the efficacy of plaque removal from the smooth surfaces is higher than that from rough surfaces. Some studies ${ }^{30,31}$ have shown that surface roughness due to subgingival SRP significantly increases subgingival microbial colonization.

A comparison of the results of different studies in this respect must be done with caution considering different methodologies and use of different measurement tools such as profilometer, Doppler laser, or electron microscope. Such differences in methodologies can explain the 
controversy in results. ${ }^{32,33}$

Within the limitations of this study, the results showed that chemical changes of periodontally-diseased root surfaces following root debridement with hand curettes with and without Er:YAG laser irradiation (100 and $150 \mathrm{~mJ}$ ) were not significantly different; although laser irradiation yielded rougher surfaces. Interestingly, Er:YAG irradiation showed higher percentages of carbon and oxygen compare to hand instrumentation that may have some clinical implications in new attachment of viable fibroblasts.

\section{Ethical Considerations}

The proposal of study was approved by the Ethics Committee, deputy of research, Shahid Beheshti University of Medical Sciences (\#9136).

\section{Conflict of Interests}

Authors declare that they have no competing interests.

\section{References}

1. Smart GJ, Wilson M, Davies EH, Kieser JB. The assessment of ultrasonic root surface debridement by determination of residual endotoxin levels. $J$ Clin Periodontol. 1990;17(3):174-178.

2. Walmsley AD, Lea SC, Landini G, Moses AJ. Advances in power driven pocket/root instrumentation. J Clin Periodontol. 2008;35(8 suppl):22-28. doi:10.1111/j.1600051X.2008.01258.x.

3. Maruyama H, Aoki A, Sasaki KM, et al. The effect of chemical and/or mechanical conditioning on the Er:YAG lasertreated root cementum: analysis of surface morphology and periodontal ligament fibroblast attachment. Lasers Surg Med. 2008;40(3):211-222. doi:10.1002/lsm.20609.

4. de Mendonca AC, Maximo MB, Rodrigues JA, Arrais CA, de Freitas PM, Duarte PM. Er:YAG Laser, ultrasonic system, and curette produce different profiles on dentine root surfaces: an in vitro study. Photomed Laser Surg. 2008;26(2):91-97. doi:10.1089/pho.2007.2129.

5. Ting CC, Fukuda M, Watanabe T, Aoki T, Sanaoka A, Noguchi T. Effects of Er,Cr:YSGG laser irradiation on the root surface: morphologic analysis and efficiency of calculus removal. J Periodontol. 2007;78(11):2156-2164. doi:10.1902/jop.2007.070160.

6. Castro GL, Gallas M, Nunez IR, Borrajo JL, Varela LG. Histological evaluation of the use of diode laser as an adjunct to traditional periodontal treatment. Photomed Laser Surg. 2006;24(1):64-68. doi:10.1089/pho.2006.24.64.

7. Theodoro LH, Haypek P, Bachmann L, et al. Effect of ER:YAG and diode laser irradiation on the root surface: morphological and thermal analysis. $J$ Periodontol. 2003;74(6):838-843. doi:10.1902/jop.2003.74.6.838.

8. Schwarz F, Aoki A, Sculean A, Georg T, Scherbaum W, Becker J. In vivo effects of an Er:YAG laser, an ultrasonic system and scaling and root planing on the biocompatibility of periodontally diseased root surfaces in cultures of human PDL fibroblasts. Lasers Surg Med. 2003;33(2):140147. doi:10.1002/lsm.10201.

9. Altundasar E, Ozcelik B, Cehreli ZC, Matsumoto K. Ultramorphological and histochemical changes after ER,CR:YSGG laser irradiation and two different irrigation regimes. J Endod. 2006;32(5):465-468. doi:10.1016/j. joen.2005.08.005.

10. Lin CP, Lee BS, Lin FH, Kok SH, Lan WH. Phase, compositional, and morphological changes of human dentin after Nd:YAG laser treatment. J Endod. 2001;27(6):389393. doi:10.1097/00004770-200106000-00004.

11. Hossain M, Kimura Y, Nakamura Y, Yamada Y, Kinoshita JI, Matsumoto K. A study on acquired acid resistance of enamel and dentin irradiated by Er,Cr:YSGG laser. J Clin Laser Med Surg. 2001;19(3):159-163. doi:10.1089/10445470152927991.

12. Hossain $M$, Nakamura $Y$, Kimura $Y$, Ito M, Yamada $Y$, Matsumoto K. Acquired acid resistance of dental hard tissues by CO2 laser irradiation. J Clin Laser Med Surg. 1999;17(5):223-226.

13. Hara AT, Ando M, Cury JA, Serra MC, Gonzalez-Cabezas $\mathrm{C}$, Zero DT. Influence of the organic matrix on root dentine erosion by citric acid. Caries Res. 2005;39(2):134-138. doi:10.1159/000083159.

14. de-Melo MA, Passos VF, Alves JJ, Barros EB, Santiago SL, Rodrigues LK. The effect of diode laser irradiation on dentin as a preventive measure against dental erosion: an in vitro study. Lasers Med Sci. 2011;26(5):615-621. doi:10.1007/s10103-010-0865-y.

15. Paula Sde S, Soares LE, do Espirito Santo AM, Martin AA, Cavalli V, Liporoni PC. FT-Raman and energy dispersive $\mathrm{X}$-ray fluorescence spectrometric analyses of enamel submitted to $38 \%$ hydrogen peroxide bleaching, an acidic beverage, and simulated brushing. Photomed Laser Surg. 2010;28(3):391-396. doi:10.1089/pho.2008.2426.

16. Folwaczny M, George G, Thiele L, Mehl A, Hickel R. Root surface roughness following Er:YAG laser irradiation at different radiation energies and working tip angulations. $J$ Clin Periodontol. 2002;29(7):598-603.

17. Kocher $\mathrm{T}$, Rosin $\mathrm{M}$, Langenbeck $\mathrm{N}$, Bernhardt $\mathrm{O}$. Subgingival polishing with a teflon-coated sonic scaler insert in comparison to conventional instruments as assessed on extracted teeth (II). Subgingival roughness. $J$ Clin Periodontol. 2001;28(8):723-729.

18. Theodoro LH, Sampaio JE, Haypek P, Bachmann L, Zezell DM, Garcia VG. Effect of Er:YAG and Diode lasers on the adhesion of blood components and on the morphology of irradiated root surfaces. J Periodontal Res. 2006;41(5):381390. doi:10.1111/j.1600-0765.2005.00846.x.

19. de Oliveira GJ, Sampaio JE, Marcantonio RA. Effects of Er,Cr:YSGG laser irradiation on root surfaces for adhesion of blood components and morphology. Photomed Laser Surg. 2010;28(6):751-756. doi:10.1089/pho.2009.2681.

20. Foroutan T, Amid R, Karimi MR. Comparison of Manual Tools, Ultrasonic and Erbium-Doped Yttrium Aluminum Garnet (Er:YAG) Laser on the Debridement Effect of the Surface of the Root of Teeth Suffering from Periodontitis. $J$ Lasers Med Sci. 2013;4(4):199-205.

21. Amid R, Kadkhodazadeh M, Fekrazad R, Hajizadeh F, Ghafoori A. Comparison of the effect of hand instruments, an ultrasonic scaler, and an erbium-doped yttrium aluminium garnet laser on root surface roughness of teeth with periodontitis: a profilometer study. $J$ Periodontal Implant Sci. 2013;43(2):101-105. doi:10.5051/ jpis.2013.43.2.101.

22. Birang R, Poursamimi J, Gutknecht N, Lampert F, Mir M. Comparative evaluation of the effects of Nd:YAG and Er:YAG laser in dentin hypersensitivity treatment. Lasers 
Med Sci. 2007;22(1):21-24. doi:10.1007/s10103-0060412-z.

23. Schwarz F, Sculean A, Berakdar M, Szathmari L, Georg T, Becker J. In vivo and in vitro effects of an Er:YAG laser, a GaAlAs diode laser, and scaling and root planing on periodontally diseased root surfaces: a comparative histologic study. Lasers Surg Med. 2003;32(5):359-366. doi:10.1002/lsm.10179.

24. Frentzen M, Braun A, Aniol D. Er:YAG laser scaling of diseased root surfaces. J Periodontol. 2002;73(5):524-530. doi:10.1902/jop.2002.73.5.524.

25. Crespi R, Barone A, Covani U. Er:YAG laser scaling of diseased root surfaces: a histologic study. J Periodontol. 2006;77(2):218-222. doi:10.1902/jop.2006.050043.

26. Tsurumaki Jdo N, Souto BH, Oliveira GJ, Sampaio JE, Marcantonio Junior E, Marcantonio RA. Effect of instrumentation using curettes, piezoelectric ultrasonic scaler and Er,Cr:YSGG laser on the morphology and adhesion of blood components on root surfaces: a SEM study. Braz Dent J. 2011;22(3):185-192.

27. Vastardis S, Yukna RA, Rice DA, Mercante D. Root surface removal and resultant surface texture with diamondcoated ultrasonic inserts: an in vitro and SEM study. $J$
Clin Periodontol. 2005;32(5):467-473. doi:10.1111/j.1600051X.2005.00705.x.

28. Blumenthal NM, Singiser RT. The enhancement of guided tissue regeneration by altering root surface topography. Int J Periodontics Restorative Dent. 1993;13(4):360-371.

29. Oberholzer R, Rateitschak KH. Root cleaning or root smoothing. An in vivo study. J Clin Periodontol. 1996;23(4):326-330.

30. Leknes KN, Lie T, Wikesjo UM, Boe OE, Selvig KA. Influence of tooth instrumentation roughness on gingival tissue reactions. J Periodontol. 1996;67(3):197-204. doi:10.1902/jop.1996.67.3.197.

31. Leknes KN, Lie T, Wikesjo UM, Bogle GC, Selvig KA. Influence of tooth instrumentation roughness on subgingival microbial colonization. J Periodontol. 1994;65(4):303-308. doi:10.1902/jop.1994.65.4.303.

32. Casarin RC, Ribeiro FV, Sallum AW, Sallum EA, NocitiJr FH, Casati MZ. Root surface defect produced by hand instruments and ultrasonic scaler with different power settings: an in vitro study. Braz Dent J. 2009;20(1):58-63.

33. Kocher $\mathrm{T}$, Langenbeck $\mathrm{N}$, Rosin $\mathrm{M}$, Bernhardt $\mathrm{O}$. Methodology of three-dimensional determination of root surface roughness. J Periodontal Res. 2002;37(2):125-131. 